that so few physicians have seen the transition and that none has reported progression of virologically proved myocarditis to dilated cardiomyopathy. In 1968 Somerville spoke of four cases of dilated cardiomyopathy which might have followed myocarditis, but a virus origin of the myocarditis had not been proved. ${ }^{8}$ There may be inflammatory cells in the myocardium in acute myocarditis, but in chronic cardiomyopathy such cells are absent. No evidence of infection with a virus or other agent can be found in the chronic disease, and virus has never been isolated from the myocardium of a patient with a chronic cardiomyopathy. There are great problems in using immunological techniques to demonstrate a virus origin: human myocardium binds gamma globulin with great avidity, and it is easy to obtain false positives when immunofluorescent techniques are used.

One of the reasons why an infective origin is often postulated is that dilated cardiomyopathy is an asymptomatic condition until the left atrial pressure rises and the patient becomes breathless. The onset of left ventricular failure in a previously fit person in whom heart disease seems unlikely is often mistaken for an upper respiratory infection. Furthermore, patients are only too anxious to believe that their symptoms date from "influenza", when the milestone was really the onset of symptomatic heart failure. Continuing bafflement surrounding the origin of dilated cardiomyopathy encourages the grasping of such straws. Talk of "conditioning factors" is another cloak for ignorance. Intercurrent infections, toxic agents, steroids, trauma, alcohol, and pregnancy (the latter in relation to puerperal cardiomyopathy) have all been so invoked.

Reduction of contractile ability with failure of ejection, consequent increase in volume, and progressive dilatation, is likely to be the indistinguishable end-result of any diffuse myocardial disorder. Dilated cardiomyopathy has no specific treatment and it is not known why the heart muscle does not contract normally. The appearances on histology and electromicroscopy are much better than the performance of the heart muscle would suggest. Endomyocardial biopsy is safe and is now eminently justified for the further investigation both of acute myocarditis and of chronic cardiomyopathy. New techniques include refined virus studies as well as immunological, metabolic, and biochemical investigations on subcellular organelles isolated by cell fractionization techniques. Using these methods, the modes and loci of abnormality may be identified and eventually the causes, when prevention and treatment might follow.

\footnotetext{
1 Abelmann, W., Circulation, 1971, 44, 950.

2 Lewes, D., Rainford, D. J., and Lane, W. F., British Heart Fournal, 1974, 36, 924 .

3 Rosenberg, H. S., and McNamara, D. G., Progress in Cardiovascular Disease, 1964, 7, 179.

4 Hirschmann, S. Z., and Hammer, G. S., American fournal of Cardiology, $1974,34,224$.

5 Grist, N. R., and Bell, E. J., Fournal of Hygiene, 1974, 73, 165.

6 Burch, G. E., et al., American Heart fournal, 1967, 74, 13.

7 Gore, I., and Saphir, O., American Heart fournal, 1947, 34, 827.

8 Somerville, W., Postgraduate Medical Fournal, 1972, 48, 746.

9 Gerzen, P., et al., British Heart Fournal, 1972, 34, 575.

10 Bengtsson, E., and Lamberger, B., American Heart fournal, 1966, 72, 751.

11 British Medical fournal (Editorial), 1972, 3, 783.
}

\section{Sézary Syndrome}

The association of erythroderma with the presence of abnormal mononuclear cells in the dermis and the peripheral blood was first described ${ }^{1}$ by Sézary and Bouvrain in 1938. For many years these characteristic mononuclear cells were thought to be reticuloendothelial in origin, and the condition was often described as a "reticulosis." There was, however, no proof to support the histiocytic nature attributed to Sézary cells.

The main clinical findings in the syndrome are generalized exfoliative erythroderma and intense pruritus: Sézary cells are present in variable numbers (often less than $10 \%$ ) in peripheral blood films. The bone marrow is spared as a rule, even in the presence of a marked lymphocytosis in the peripheral blood. Other investigations such as the E.S.R., lymphangiography, and the immunological profile are often normal. Two types of Sézary cells have been recognized: a large cell which corresponds to the cell with a convoluted or cerebriform nuclear appearance seen by electron microscopy, and a small cell which may resemble a normal lymphocyte by light microscopy. Either type may dominate the picture, and a peripheral blood lymphocytosis is not uncommon in the small cell variant. These cases may, therefore, occasionally be misdiagnosed as chronic lymphocytic leukaemia.

A new interest in the condition arose from elegant cytogenetic studies published in 1973 which showed that the Sézary cell responds in a normal fashion to the $\mathrm{T}$-lymphoid cell mitogen phytohaemagglutinin (P.H.A.). Last year a symposium on the Sézary cell was held at the Mayo Graduate School of Medicine. ${ }^{3}$ It dealt comprehensively with the many clinical and diagnostic problems of the syndrome and provided strong evidence for the T-lymphocyte origin of the characteristic Sézary cell.

T-cell markers (formation of spontaneous rosettes with sheep erythrocytes, reactivity with anti- $T$ cell sera, and response to PHA) have been found in both variants of Sézary cells, which at the same time give negative results when tested for B-cell markers (surface-bound immunoglobulins, receptors for $\mathrm{C}_{3}$ ). The immunological, cytochemical, and ultrastructural features in the Sézary cell rule out any relationship with the mononuclear-phagocyte system of cells (monocytes and histiocytes). Cytochemically, Sézary cells show a positive acid phosphatase reaction, ${ }^{4}$ a feature also associated with other T-lymphoproliferative disorders, particularly T-lymphoblastic leukaemia ; ${ }^{5}$ this reaction may also help to distinguish Sézary cells from the lymphocytes of chronic lymphocytic leukaemia, whose acid phosphatase content is nearly always extremely low. ${ }^{4}$

According to their DNA content, which corresponds to the chromosomal number, Sézary cells may be diploid or heteroploid. ${ }^{23}$ The number of chromosomes in the heteroploid population ranges from 52 to 99 . The cytogenetic findings bear some resemblance to those observed in long-term cultures of lymphoblastoid cell lines; this and the incidence of the disease in the second half of life suggests that the chromosome aberrations observed may be related to ageing, suggesting a clonal escape of $\mathrm{T}$-cells from the mechanism of immune surveillance. ${ }^{6}$

The relation of the Sézary syndrome to other lymphomas is of great interest. A malignant lymphoma develops in $25 \%$ of cases $;^{7}$ and patients with a pre-Sézary syndrome of variable duration have been recognized. A close relation has also been suggested between the Sézary syndrome and mycosis fungoides, ${ }^{3}$ in which $\mathrm{T}$-cell markers have been found in the cells infiltrating the skin and lymph nodes.

The results of treatment have, on the whole, been disappointing. ${ }^{3}$ Remissions after conventional radiotherapy are of short duration, and vigorous combination chemotherapy is also of limited value. Good control of the disease and of its symptoms, pruritus in particular, has been obtained with alkylating agents such as chlorambucil combined with prednisone given continuously at low dosage. Promising results have 
also been achieved by whole-body surface electron therapy. ${ }^{8}$ Intensive leukopheresis by means of a continuous-flow cell separator has produced dramatic improvement of the skin lesions and the blood picture in a patient with advanced Sézary syndrome and a high lymphocyte count. ${ }^{9}$

\footnotetext{
1 Sézary, A., and Bouvrain, Y., Bulletin de la Société française de dermatologie et de syphiligraphie, 1938, 45, 254.

2 Crossen, P. E., et al., American fournal of Medicine, 1971, 50, 24

3 Symposium on the Sézary cell, Mayo Clinic Proceedings, 1974, 49, 513.

4 Flandrin, G., and Daniel, M. T., Scandinavian fournal of Haematology, 1974, 12, 23 .

1974, 12, 23.
Catovsky, D., et al., fournal of Clinical Pathology, 1974, 27, 767.

6 Prunieras, M., Mayo Clinic Proceedings, 1974, 49, 548.

7 Winkelmann, R. K., Mayo Clinic Proceedings, 1974, 49, 519.

${ }^{8}$ Szur, L., British fournal of Dermalology, 1964, 76, 10.

${ }^{9}$ Edelson, R., et al., New England fournal of Medicine, 1974, 291, 293.
}

\section{More about Febrile Convulsions}

The management of so-called febrile convulsions varies as much as ideas upon their pathogenesis. At one extreme are those who maintain that brief seizures in a 2-year old with a feverish cold are invariably benign and can in the main be disregarded, while ranged against this view are those who believe that febrile convulsions are within the spectrum of epilepsy and should be managed according to exactly the same principies. Practice based on the one view might affirm the need to control pyrexia and treat an infection, while those motivated by anxiety about epilepsy might recommend continuous anticonvulsant administration not only to the child who has had a fit ${ }^{2}$ but also to his preschool siblings, because of the familial tendency.

Clearly these differences in practice depend on whether febrile convulsions are of themselves thought to be damaging and potential precursors of more chronic seizure states. Unfortunately, some retrospective studies intended to delineate their natural history have embodied circular arguments inherent in the definition of febrile convulsions. ${ }^{134}$ The bestknown English researches are those of Ounstead et al. at Oxford, ${ }^{5}$ which suggested genetic and pathogenetic relationships between fever-associated fits and more chronic seizure states, especially temporal-lobe epilepsy in later childhood.

Such studies lead to the conclusion that there is an agedependent inherited tendency which may become expressed as a convulsion under a particular stress. Most paediatricians accept the importance of fever as such-either through its rate of increase ${ }^{6}$ or its absolute level ${ }^{7}{ }^{8}$ - but in fact the evidence that fever rather than the infection is responsible is far from convincing. It could even be argued that stress (or rather distress) should be more widely defined to include not only infection, ${ }^{9}$ and perhaps fever and metabolic changes, ${ }^{2}$ but also teething, since the evidence for infection in every case is unconvincing and transient fever is a concomitant of most fits, however caused. Though such fits are usually trivial if they are severe and prolonged they may be fatal or if not may cause permanent structural damage to the brain, including so-called Ammon's horn sclerosis ${ }^{10} 11$ or mesial temporal sclerosis, ${ }^{12} 13$ thought possibly to be the structural basis of many cases of temporal lobe epilepsy. Implicit in this hypothesis is the belief that this outcome can be prevented by appropriate therapeutic intervention during the period of maximum risk, from 1 to 4 years.
Should, then, one commit all children who have had a single febrile fit to continuous anticonvulsant proplylaxis as well as to occasional antipyretics? The issue would be less important if the effect of anticonvulsants, particularly barbiturates and hydantoins, were an unmitigated blessing. Effective prevention of febrile fits requires medication to be given regularly and not intermittently ${ }^{1415}$ and barbiturates are more effective than hydantoin ${ }^{14}{ }^{16}{ }^{17}$. But British paediatricians acknowledge that three quarters or more of young children show unfavourable behavioural changes on phenobarbitone, while gum hypertrophy, hirsutism, and a suspicion that there may be interference with normal cerebellar development in the very young ${ }^{18}$ discourage the use of phenytoin. Worse still is the anxiety that anticonvulsants may interfere with learning in some children. ${ }^{19}$

Since at worst, irrespective of management, only 5 to $10 \%$ of children who present with supposed febrile convulsions will graduate to more chronic seizure disturbance, ${ }^{2}$ the matter of continued drug prophylaxis needs to be weighed carefully. It would be helpful if children at especially high risk of sequelae could be recognized, and Wallace has recently claimed to have identified certain high risk groups. ${ }^{20}$ The fact that it was a hospital-based study may have accounted for the high rate of recurrence $(47 \%)$ but this is unlikely to affect the other conclusions. There was an increased risk of recurrence in boys with a positive family history of any form of seizure among first-degree relatives (19/29); girls in whom the first fit occurred before 19 months (17/26); children of either sex whose first fit was prolonged $(36 / 60)$; those with persisting neurological abnormality (38/68). The data are of sufficient predictive value to support a policy of prophylaxis against damaging seizures only in the first two groups, since the others probably comprised a high proportion of children with epileptogenic lesions anyway. Moreover, the obverse groups did not have sufficiently low recurrence rates necessarily to deny them the potential benefits of prophylaxis: 15 of 43 boys without a family history had another convulsion, while 4 of 18 girls whose first fit occurred after 19 months also had another fit. It should be stressed that this was not a study of epilepsy in the usually accepted sense but of recurrence (sometimes single) of febrile fits. Longer term and more extensive studies will be required not only to delineate more clearly risk factors for epilepsy itself but also to show that drug prophylaxis can prevent its occurrence.

In the absence of better discrimination a treatment policy should try to balance the established hazard of uncontrolled febrile convulsions against the disadvantages of prolonged and unnecessary treatment with drugs known to have unpleasant side effects. Any convulsion that lasts longer than 15 to 20 minutes warrants treatment with parenteral anticonvulsants (phenobarbitone, diazepam, or paraldehyde); accompanying high fever may need tepid sponging as well as aspirin, which can be given by suppository.

For prophylaxis, in addition to antipyretics as necessary, regular medication with phenobarbitone or phenytoin (both in a dosage of $5-7 \mathrm{mg} / \mathrm{kg}$ body weight daily), whichever is tolerated better, can be offered to those children whose first fit has been severe or has left a neurological deficit and to those who have had two or more fits. For the child who has had only one brief fit, advice on tepid sponging and antipyretics is all that is needed and should prove effective in protecting against further attacks in most cases.

1 British Medical fournal, 1972, 2, 608.

Lennox-Buchthal, M. A., Electroencephalography and Clinical Neurophysiology, 1973. Supplement 32. 